Communications in Physics, Vol. 24, No. 2 (2014), pp. 124-134

\title{
THE PHASE TRANSITION OF HALF-SKYRMIONS IN THE DENSE NUCLEAR MATTER ${ }^{1}$
}

\author{
NGUYEN AI VIET \\ Information Technology Institute, Vietnam National University, Hanoi, Vietnam
}

Received 15 March 2014

Accepted for publication 10 May 2014

E-mail: naviet@vnu.edu.vn

\begin{abstract}
In this paper we have discussed all possible boundary values used in the ordinary Skyrme model. Among the solutions to these boundary conditions the non-skyrmion and skyrmion one exist according to certain choices. The free parameter $R$ in the boundary value conditions is shown to be related to the density of the nuclear matter in its Wigner-Seitz model. All three types of skyrmion can describe the nucleon in a good agreement with the experimental values of the axial coupling constant and isoscalar radii. However, all three types of skyrmion lead to a large value of the nucleon mass and a too small mass splitting between the nucleon and delta particles. The phase transitions of the skyrmions of Type III are also observed in the numerical computations as the density of the nuclear matter varies.
\end{abstract}

Keywords: quantum field theories, nonlinear chiral model, Skyrme model, computational physics, dense nuclear matter.

\section{INTRODUCTION}

It was Skyrme who has shown long ago for the first time that nucleons can be treated as solitons in the non-linear sigma model [1]. The interest toward the Skyrme model has revived after Adkins, Nappi and Witten [2] have successfully derived the static properties of nucleons from the model within $30 \%$ of the experimental values. However, if the calculations are carried out more precisely, the model has two main problems. First, since the pion mass is taken as zero, one can prove that the mass splitting between nucleon and delta is zero. The non-zero result is obtained only if one truncate the large distant contribution of a divergent integral. The lesson learned from this problem is that one must be more careful with the numerical analysis. The introduction of non-zero pion mass could have fixed this problem [3]. However, it made the worse predictions of the static properties of nucleons. The second problem of the traditional Skyrme model is that if one uses the experimental values of the model parameters like the pion decay constant $F_{\pi}=186 \mathrm{MeV}$ and the pion mass $m_{\pi}=138 \mathrm{MeV}$ as input, the predictions for the static properties of nucleon do not look as good as presented in $[2,3]$, when the nucleon and delta masses are used as inputs. In the recent works $[4,5]$, these problems have been reviewed and reexamined more carefully with new

\footnotetext{
${ }^{1}$ Based on the talk given at the the 38th National Conference on Theoretical Physics, Da Nang, August 2013.
} 
numerical combined with a global analysis. These works also proposed new solutions constrained within a finite radius, which might describe nucleons in the dense nuclear matter.

This paper presents the numerical results about the possible phase transition of different baryon numbered solutions when the density varies. Actually, this phenomena is in harmony to the chain of phase transitions occurs to the strong interacting matter: At the high temperature, the quark gluon matter is rare and described by QCD in asymptotic freedom phase. In this phase quark has almost zero mass, so QCD has an inherent linear chiral symmetry $S U(2) \times S U(2)$. As the matter cools down, the matter is denser and the transition to the pion condensate phase occurs. In this phase, the quark acquires a mass and the chiral symmetry is broken spontaneously and realized non linearly. The effective theory in this phase is the non-linear sigma model of pion degree of freedom. As the matter continues to cool down, the model evolves to the Skyrme one and the nucleons emerge as solitons. When nuclear matter is so dense as inside the neutron stars, the skyrmions are compressed to a finite size and the high baryon numbered skyrmions can emerge.

The numerically computed static properties of these new skyrmions in the original Skyrme model do not agree too well with the experimental values. However, since only few first terms of the effective meson theory are known from the experimental data, in the future works, one can modify the higher order terms in the original Skyrme model to improve the predictions in the modified Skyrme models [6,7] or in the extended Skyrme models [8,9].

The paper is organized as follows: Sect.II. gives a brief overview about the boundary condition problems of the Skyrme model. The new skyrmions, which are restricted in a finite sphere of radius $R$, are also suggested. The values of $\mathrm{R}$ are shown to be related to the nuclear density. In Sec. III, the numerically computed solutions of type III are also presented. The phase transitions between different topological charge solutions of type III are observed at various values of the control parameter $m$. Sec. IV summarizes the results and discusses the possible applications.

\section{THE MODEL}

\section{II.1. Generalities}

The original Skyrme model with the massive pion [4] is the simplest effective theory of the nonlinear pion field

$$
U(\vec{x}, t)=\exp \left(i \pi_{a}(\vec{x}, t) \sigma^{a}\right)
$$

where $U(\vec{x}, t)$ is a $S U(2)$ matrix function, $\pi_{a}(\vec{x}, t)$ and $\sigma^{a}$ are the triplets of pion fields and Pauli matrices.

The basic element to construct the model is $U^{\dagger} \partial_{\mu} U$, which used to be written as a sum of two elements as follows

$$
U^{\dagger} \partial_{\mu} U=i\left(\mathcal{D}_{\mu} \pi^{a}+\Gamma_{\mu}^{a}\right) \sigma^{a}
$$

where

$$
\begin{aligned}
\mathcal{D}_{\mu} \pi^{a}=\frac{\vec{\pi} \partial_{\mu} \vec{\pi}}{\pi^{2}} \pi^{a}+\frac{\sin (2 \pi)}{2 \pi}\left(\partial_{\mu} \pi^{a}-\frac{\vec{\pi} \partial_{\mu} \vec{\pi}}{\pi^{2}} \pi^{a}\right), \\
\Gamma_{\mu}^{a}=\frac{\sin ^{2}(\pi)}{\pi^{2}} \epsilon^{a b c} \partial_{\mu} \pi_{b} \pi_{c} \\
\pi=\pi(\vec{x}, t)=\sqrt{\pi^{a} \pi_{a}}
\end{aligned}
$$


The Lagrangian governing the model is as follows

$$
\begin{aligned}
\mathcal{L}_{S K}= & \frac{F_{\pi}^{2}}{16} \operatorname{Tr}\left(\partial_{\mu} U \partial^{\mu} U^{\dagger}\right)+\frac{1}{32 e^{2}} \operatorname{Tr}\left(\left[\partial_{\mu} U U^{\dagger}, \partial_{\nu} U U^{\dagger}\right]^{2}\right)+\frac{F_{\pi}^{2} m_{\pi}^{2}}{8} \operatorname{Tr}(U-1) \\
= & \frac{F_{\pi}^{2}}{8}\left(\mathcal{D}_{\mu} \vec{\pi}+\vec{\Gamma}_{\mu}\right) \cdot\left(\mathcal{D}^{\mu} \vec{\pi}+\vec{\Gamma}_{\mu}\right)+\frac{1}{16 e^{2}}\left[\left(\mathcal{D}_{\mu} \vec{\pi}+\vec{\Gamma}_{\mu}\right) \times\left(\mathcal{D}_{\nu} \vec{\pi}+\vec{\Gamma}_{\nu}\right)\right]^{2} \\
& \left.+\frac{F_{\pi}^{2} m_{\pi}^{2}}{8} \cos (\pi(\vec{x}, t))-1\right),
\end{aligned}
$$

where the pion decay constant $F_{\pi}=186 \mathrm{MeV}$ and the pion mass $m_{\pi}=138 \mathrm{MeV}$. The parameter $e$ must satisfy the Balachandran's bound [10]

$$
1.57<e<4.17
$$

to be consistent. The spherically symmetric skyrmions are constructed from the hedge hog ansatz as follows

$$
\pi^{a}=F(r) x^{a},
$$

where $\infty>r=F_{\pi} e \rho \geq 0$ is a dimensionless variable, which is used instead of the radial coordinate $\rho$ in the polar reference frame $(\rho, \phi, \theta) . F(r)$ is a real value function. The classical energy is derived from the Lagrangian (5) as follows

$$
\begin{aligned}
M= & \frac{4 \pi F_{\pi}}{e} M_{0}=\frac{4 \pi F_{\pi}}{e} \int_{0}^{R}\left(\frac{\sin ^{2}(F(r))}{4}+\frac{\left(r F^{\prime}(r)\right)^{2}}{8}+\frac{m^{2} r^{2}}{4}(1-\cos (F(r)))\right. \\
& \left.+\sin ^{2}(2 F(r))\left[\frac{\sin ^{2}(F(r))}{2 r^{2}}+\left(F^{\prime}(r)\right)^{2}\right]\right) .
\end{aligned}
$$

The profile functions $F(r)$, which minimize the classical energy in the formula (8) are solutions of the following equation of motion:

$$
\begin{aligned}
F^{\prime \prime}(r)\left(2 \sin ^{2}(F(r))+\frac{r^{2}}{4}\right)= & \sin (2 F(r))\left(-\left(F^{\prime}(r)\right)^{2}+\frac{\sin ^{2}(F(r))}{r^{2}}+\frac{1}{4}\right) \\
& -\frac{r F^{\prime}(r)}{2}+\frac{m^{2} r^{2}}{4} \sin (F(r)) .
\end{aligned}
$$

Instead of the pion mass $m_{\pi}$, the following dimensionless parameter is used

$$
m=\frac{m_{\pi}}{e F_{\pi}}
$$

Since the experimental values of $F_{\pi}$ and $m_{\pi}$ are known, the model has only the free parameter $m$ to fit the theoretical predictions with the experiments. The parameter $m$ must satisfy the Balachandran's bound condition (6) as follows

$$
0.18<m<0.48
$$

In order to solve Eq. (9), one must have a boundary or initial condition. Such a condition is determined from the topological charge conservation. The model (5) has the following conserved topological current

$$
B^{\mu}=\frac{\epsilon^{\mu \nu \rho \tau}}{24 \pi^{2}} \operatorname{Tr}\left(U^{\dagger} \partial_{\nu} U U^{\dagger} \partial_{\rho} U U^{\dagger} \partial_{\tau} U\right) .
$$


The conserved topological charge derived from Eq. (12) in the hedge hog ansatz is given as follows

$$
B^{0}=-\frac{2}{\pi} \int_{F(0)}^{F(R)} \sin ^{2}(F(r)) d F=\frac{F(0)-F(R)}{\pi}=N
$$

The solutions with the topological charge $B^{0}=N$ must satisfy the following condition

$$
F(0)-F(R)=N \pi \text {. }
$$

\section{II.2. Boundary conditions and solutions}

In our recent works [4,5], from the asymptotic behavior of the Skyrme equation(9) near the origin $r=0$, we have shown that the possible boundary conditions at $r=0$ must be as follows

$$
F(0)=M \frac{\pi}{2}
$$

where $M$ is an arbitrary integer. It has been also shown [4,5] that the skyrmion solution with $M=2 n$ have a finite energy, and the non-skyrmion solutions with $M=2 n+1$ have an infinite energy. So, beside the traditional infinite range skyrmions of Type I, we have the following two types of skyrmions with the finite range $R$ :

i) The skyrmions of Type II satisfy the boundary condition

$$
F(0)=n \pi ; F(R)=k \frac{\pi}{2}
$$

where $n$ and $k$ are integers. From Eq.(13), we can see that the topological charge is $N=n-k / 2$, which can be an integer or half-integer.

ii) The skyrmions of Type III satisfy the boundary conditions

$$
F(0)=n \pi ; F(R)=k \frac{\pi}{2} ; F^{\prime}(R)=0
$$

In this case, if we choose an even integer $k=2 m$, the initial conditions $F^{\prime}(R)=0$ and $F(R)=$ $m \pi$ together with the equation of motion (9) imply that all the higher derivatives vanish at $r=R$. Hence, $F(r)=m \pi$ is a constant valued function. In other words, the skyrmions of Type III exist only with an odd value of $k=2 m+1$. The topological charge of the skyrmions of Type III is always a half-integer number $N=(n-m)-1 / 2$.

\section{II.3. Non-skyrmion solutions and the stability of skyrmions}

The non-skyrmion solutions with non-finite energy satisfies the boundary condition

$$
F(0)=(2 n+1) \frac{\pi}{2} \text {. }
$$

So, between the non-skyrmion solutions going to $F(0)=(2 n+1) \frac{\pi}{2}$ and $F(0)=(2 n-1) \frac{\pi}{2}$ there is a skyrmion solution going to $F(0)=n \pi$. The numerical computations [4,5] have shown that the non-skyrmion solution trajectories are so dense and there is only one skyrmion solution trajectory separating between to groups of non-skyrmion solutions. This energetically minimal behavior guarantees the stability of the skyrmion solution.

From the topological point of view, the stability of the skyrmions is guaranteed by the conservation of the integer topological charge. However, as we have seen, new skyrmions can have a half-integer topological charge, which can not be interpreted as a winding number as in the traditional case. 


\section{II.4. Half-skyrmions}

The skyrmions of Type II can have either integer or half-integer topological charge, while the skyrmions of Type III can have only half-integer topological charge. The half-integer topological charged skyrmions, which can be called as half-skyrmions, have been discovered firstly by Goldhaber and Manton [11]. Recently, the half-skyrmions have attracted attentions to their existence in the high density hadronic matter inside the compact stars [12] and in the strongly correlated planar copper systems, which are relevant to the high- $T_{c}$ superconductivity.

Our half-skyrmions are obtained in a different context due to the restriction the skyrmions within the finite sized domain. Mathematically speaking, the half-integer topological number emerges when the half line $[0, \infty]$ is replaced by the interval $[0, R]$. The understanding of this phenomena on the mathematical basis is still open for us.

\section{NEW SKYRMIONS AND DENSE NUCLEAR MATTER}

\section{III.1. Dense nuclear matter modeling}

The solutions of the Skyrme model with the crystalline symmetries have been also investigated long ago [14] and become widely used in modelling the nuclear and high- $T_{c}$ superconductivity matter. On the other hand, one can also use the famous Wigner-Seitz approximation to model a large crystalline material by a single cell, if we know the solution in one cell. With new solutions at hand, we can use the Wigner-Seitz approximation to build a crystalline material from the new finite range skyrmions, which can be a model for the dense nuclear matter.

Since the physical properties of the many body systems are sensitive with the ones of a single body, the static properties of skyrmions must be fitted with their experimental values of nucleons. If necessary, the Skyrme model must be modified to have the best experimental fit. Since, only first terms of the Skyrme model are fixed by the experiments, one can always modify the model by adding higher terms [6-9], in order to have better theoretical predictions.

\section{III.2. Static properties of skyrmions in the Skyrme model}

The traditional skyrmions of Type I are known to model the nucleons in the massless pion case within $30 \%$ error. However, the calculations in this case have suffered from the unjustified truncation of a divergent integrand [4]. The introduction of the pion mass has fixed this divergence problem, but it has given worse predictions.

In [4] we have introduced a new fitting schema, which can give a more objective evaluation about the predictive power of the model. The new schema uses the model parameters $F_{\pi}, m_{\pi}$ and the free parameter $m$ as inputs. The nucleon and delta masses are now outputs of the model.

The static properties of skyrmion of Type I have been computed based on the same formulas of $[2,3]$ in the new fitting schema and listed in Table 1

In the above table we can see two different intervals of the free parameter $m$ have brought different fitting results: Between the Balachandran's bound $0.48>m>0.18$, the coupling constant $g_{A}$ and other radii can be fitted very well with a extremely high accuracy. However, in the same interval, the mass of nucleon is between 2-5 times of the experimental value $m_{N}=939 \mathrm{MeV}$ or at best $100 \%$ error, while the mass difference between nucleon and delta is zero. In the region $m<0.11$ the nucleon mass or the mass difference between the nucleon and delta can be fitted with the experimental values, but the other static properties strongly disagree with experiments. 
Table 1. The static properties of baryons as the skyrmions of Type I

\begin{tabular}{|c|c|c|c|c|c|c|c|}
\hline $\begin{array}{c}\text { Static } \\
\text { Properties }\end{array}$ & $\begin{array}{c}\mathbf{m}= \\
\mathbf{0 . 4 8}\end{array}$ & $\begin{array}{c}\mathbf{m}= \\
\mathbf{0 . 1 8}\end{array}$ & $\begin{array}{c}\mathbf{m}= \\
\mathbf{0 . 1 3 2 1}\end{array}$ & $\begin{array}{c}\mathbf{m}= \\
\mathbf{0 . 1 0 1 4}\end{array}$ & $\begin{array}{c}\mathbf{m}= \\
\mathbf{0 . 0 0 5 1 2 5}\end{array}$ & $\begin{array}{c}\mathbf{m}= \\
\mathbf{0 . 0 0 2 1 6 7}\end{array}$ & $\begin{array}{c}\text { Experimental } \\
\text { Values }\end{array}$ \\
\hline$e$ & 1.55 & 4.12 & 5.616 & 7.317 & 145 & 342 & $1.57<\mathrm{e}<4.17$ \\
\hline$M^{s=\frac{1}{2}}$ & $5012 \mathrm{MeV}$ & $1702 \mathrm{MeV}$ & $1232 \mathrm{MeV}$ & $939 \mathrm{MeV}$ & $120 \mathrm{MeV}$ & $939 \mathrm{MeV}$ & $939 \mathrm{MeV}$ \\
\hline$M^{s=\frac{3}{2}}-M^{s=\frac{1}{2}}$ & 0 & 0 & 0 & 0 & $293 \mathrm{MeV}$ & $3676 \mathrm{MeV}$ & $293 \mathrm{MeV}$ \\
\hline$r_{B}^{I=0}$ & $1.08 \mathrm{fm}$ & $0.495 \mathrm{fm}$ & $0.376 \mathrm{fm}$ & $0.295 \mathrm{fm}$ & $0.0155 \mathrm{fm}$ & $0.066 \mathrm{fm}$ & $0.72 \mathrm{fm}$ \\
\hline$r_{B, m}^{I=0}$ & $1.46 \mathrm{fm}$ & $0.71 \mathrm{fm}$ & $0.549 \mathrm{fm}$ & $0.436 \mathrm{fm}$ & $0.0242 \mathrm{fm}$ & $0.010 \mathrm{fm}$ & $0.81 \mathrm{fm}$ \\
\hline$g_{A}$ & 2.65 & 0.456 & 0.255 & 0.154 & $7.76 \times 10^{-5}$ & $<0$ & 1.23 \\
\hline
\end{tabular}

The static properties of the skyrmions of Type II with the topological charge $N=1$ with at various values of $R$ in Table 2 .

Table 2. Static properties of the skyrmions of Type II with $\mathrm{m}=0.273$

\begin{tabular}{|c|c|c|c|c|c|}
\hline $\boldsymbol{R}$ & $M_{N}$ & $\mathbf{M}_{\boldsymbol{\Delta}}-\mathbf{M}_{\mathbf{N}}$ & $\mathbf{r}_{\mathbf{B}}^{\mathbf{I}=\mathbf{0}}$ & $\mathbf{r}_{\mathbf{B}, \mathbf{m}}^{\mathbf{I}=\mathbf{0}}$ & $\mathbf{g}_{\mathbf{A}}$ \\
\hline $\mathrm{N} / \mathrm{A}$ & $939 \mathrm{MeV}$ & $293 \mathrm{MeV}$ & $0.72 \mathrm{fm}$ & $0.81 \mathrm{fm}$ & 1.23 \\
\hline$\infty$ & $2648 \mathrm{MeV}$ & $0.0035 \mathrm{MeV}$ & $0.70 \mathrm{fm}$ & $0.98 \mathrm{fm}$ & 0.98 \\
\hline 10 & $2661 \mathrm{MeV}$ & $0.0036 \mathrm{MeV}$ & $0.70 \mathrm{fm}$ & $0.97 \mathrm{fm}$ & 0.97 \\
\hline 5 & $2726 \mathrm{MeV}$ & $0.0043 \mathrm{MeV}$ & $0.65 \mathrm{fm}$ & $0.86 \mathrm{fm}$ & 0.88 \\
\hline 3.5 & $2875 \mathrm{MeV}$ & $0.0051 \mathrm{MeV}$ & $0.57 \mathrm{fm}$ & $0.72 \mathrm{fm}$ & 0.75 \\
\hline 2.7 & $3088 \mathrm{MeV}$ & $0.0061 \mathrm{MeV}$ & $0.49 \mathrm{fm}$ & $0.61 \mathrm{fm}$ & 0.64 \\
\hline 1.7 & $3800 \mathrm{MeV}$ & $0.0084 \mathrm{MeV}$ & $0.35 \mathrm{fm}$ & $0.43 \mathrm{fm}$ & 0.45 \\
\hline 1.2 & $4740 \mathrm{MeV}$ & $0.011 \mathrm{MeV}$ & $0.27 \mathrm{fm}$ & $0.32 \mathrm{fm}$ & 0.33 \\
\hline
\end{tabular}

We observed that if we choose the free parameter $m=0.273$, which is within the Balachandran's bound, we can fit the coupling $g_{A}$ and the radii with a high accuracy but the mass of nucleon is triple of the experimental value. So if we choose the skyrmions of Type II as a model of nucleon, the error of the theoretical prediction for the nucleon mass is as high as $200 \%$.

The static properties of the skyrmions of Type III with a topological charge $N=1 / 2$ are listed in Table 3 at various values of $m$. Let us note that if we want to model the baryons with half-skyrmions, we will have to redefine the baryon charge as twice of the topological one.

Table 3. Static properties of the skyrmions of Type III with $\mathrm{N}=1 / 2$

\begin{tabular}{|c|c|c|c|c|c|c|}
\hline Static Properties & $m=0.48$ & $m=0.273$ & $m=0.18$ & $m=0.1$ & $m=0.05$ & $m=0.01$ \\
\hline$e$ & 1.55 & 2.72 & 4.12 & 7.42 & 14.84 & 74.19 \\
\hline$M_{s=\frac{1}{2}}$ & $4963 \mathrm{MeV}$ & $5556 \mathrm{MeV}$ & $3504 \mathrm{MeV}$ & $1898 \mathrm{MeV}$ & $941 \mathrm{MeV}$ & $199 \mathrm{MeV}$ \\
\hline$M_{s=\frac{3}{2}}-M_{s=\frac{1}{2}}$ & $0.0005 \mathrm{MeV}$ & $0.002 \mathrm{MeV}$ & $0.008 \mathrm{MeV}$ & $0.047 \mathrm{MeV}$ & $0.375 \mathrm{MeV}$ & $47 \mathrm{MeV}$ \\
\hline$r_{B}^{I=0}$ & $1.162 \mathrm{fm}$ & $0.382 \mathrm{fm}$ & $0.166 \mathrm{fm}$ & $0.051 \mathrm{fm}$ & $0.013 \mathrm{fm}$ & $0.51 \times 10^{-3}$ \\
\hline$r_{B, m}^{I=0}$ & $2.004 \mathrm{fm}$ & $1.139 \mathrm{fm}$ & $0.751 \mathrm{fm}$ & $0.417 \mathrm{fm}$ & $0.208 \mathrm{fm}$ & $41.2 \times 10^{-3}$ \\
\hline$g_{A}$ & 2.881 & 0.343 & 0.065 & 0.006 & 0.0004 & $6.14 \times 10^{-7}$ \\
\hline
\end{tabular}

We can see that the experimental fit is not too optimistic in this case, if one want to model the nucleon by the skyrmion of Type III. 
We can conclude that the skyrmions of the Skyrme model cannot describe nucleons within an error of even $100 \%$. This result is a little bit disappointing, however, it paves the way to the various modifications of the Skyrme model. In particular, such a model like in Ref.[6], which predicts the low nucleon mass in the old fitting schema, will potentially have a good experimental fit.

\section{III.3. Nuclear density and the skyrmion's range parameter}

The radius $\mathrm{R}$ of the new skyrmions of Type II and Type III must have a physical meaning to have phenomenological implications. In fact, we are able to establish a relationship between the value of $\mathrm{R}$ and the nuclear matter density. We will follow the nuclear matter density patterns of P.Hansen et al. [10] and find the values of R corresponding to those patterns.

First, let us discuss about the units of the parameters to be used in this paper. The input parameters $F_{\pi}$ and $m_{\pi}$ of the Skyrme model are measured in $\mathrm{MeV}$. On the other hand, the radii and distant in dense nuclear matter are measured in fm (fermi). The conversion between $\mathrm{MeV}$ and $f m$ is always possible by using the conversion constant $197.33 \mathrm{MeV} \times f m=1$. In this paper, the dimensionless variable $r=F_{\pi} e \rho$ is used, where the radial coordinate $\rho$ is measured in $\mathrm{fm}$. The nucleon baryon radius $\rho_{N 0}=0.72 \mathrm{fm}$, where $1 \mathrm{fm}=10^{-15} \mathrm{~cm}=1 / 197.33 \mathrm{MeV}^{-1}$. Hence the dimensionless radius of nucleon $r_{N 0}$ can be expressed as follows

$$
r_{N 0}=F_{\pi} e \rho_{N 0}=0.69 e
$$

Applying the Balachandran's bound (6), we obtain the following bound for the dimensionless radius of nucleon

$$
1.07<r_{N 0}<3.87
$$

From Eq. (19) we obtain the following ratio

$$
\frac{e}{r_{N 0}}=1.45
$$

The volume of the nucleon as a sphere of radius $r_{N 0}$ is as follows

$$
V_{N 0}=\frac{4}{3} \pi \rho_{N 0}^{3}=\frac{4}{3} \pi\left(\frac{r_{N 0}}{F_{\pi} e}\right)^{3} .
$$

The saturated baryon density of nuclear matter $\varrho_{N 0}$ is where nucleons begin to touch. That will be different from the density in the volume $V_{N 0}$ by a factor of $\frac{\pi}{3 \sqrt{2}}$, because it is the density of the equal nucleon spheres closed-packed

$$
\varrho_{N 0}=\frac{\pi}{3 \sqrt{2}} \frac{1}{V_{N 0}}=\frac{1}{4 \sqrt{2}}\left(\frac{F_{\pi} e}{r_{N 0}}\right)^{3}=0.148\left(\frac{e}{r_{N 0}}\right)^{3}=0.47\left(\mathrm{fm}^{-3}\right)
$$

The baryon density of the skyrmions of Types II and III can be calculated as follows

$$
\varrho_{N R}=\frac{1}{4 \sqrt{2}}\left(\frac{F_{\pi} e}{R}\right)^{3}=0.148\left(\frac{e}{R}\right)^{3}
$$

Comparing Eqs. (23) and (24), we obtain the ratio of the baryon density of the given nuclear matter to the saturated nuclear density as follows

$$
\frac{\varrho_{N R}}{\varrho_{N 0}}=\left(\frac{r_{N 0}}{R}\right)^{3}
$$


which can be related to the value of $R$ as follows

$$
R=r_{N 0}\left(\frac{\varrho_{N 0}}{\varrho_{N R}}\right)^{\frac{1}{3}}=\frac{F_{\pi} e}{197.33} 0.72\left(\frac{\varrho_{N 0}}{\varrho_{N R}}\right)^{\frac{1}{3}}=\frac{0.5045}{m}\left(\frac{\varrho_{N 0)}}{\varrho_{N R}}\right)^{\frac{1}{3}}
$$

P.Haensel et al. [10] have classified the states of the nuclear matter which are equivalent to the ones in neutron stars. Using Eq.(25), the values of $\mathrm{R}$ can be classified according to the corresponding nuclear density as follows:

i) The nuclear matter state in the inner core of neutron stars: $0.94<\varrho_{N R}<7.052\left(\left(\mathrm{fm}^{-3}\right)\right)$. The corresponding range of $\mathrm{R}$ at various values of $\mathrm{m}$ are listed in Table 4 .

Table 4. The ranges of $R$ corresponding to the inner core of neutron stars

\begin{tabular}{|c|c|c|c|}
\hline $\boldsymbol{m}$ & 0.48 & 0.273 & 0.18 \\
\hline $\boldsymbol{R}$ & $0.4265<R<0.8342$ & $0.7493<R<1.4668$ & $1.2364<R<2.2256$ \\
\hline
\end{tabular}

ii) The nuclear matter state of the outer core of neutron stars: $0.235<\varrho_{N R}<0.94\left(\left(\mathrm{fm}^{-3}\right)\right)$. The corresponding range of $\mathrm{R}$ at various values of $\mathrm{m}$ are listed in Table 5.

Table 5. The ranges of $\mathrm{R}$ corresponding to the outer core of neutron stars

\begin{tabular}{|c|c|c|c|}
\hline $\mathbf{m}$ & 0.48 & 0.273 & 0.18 \\
\hline $\mathbf{R}$ & $0.8342<R<1.3243$ & $1.4668<R<2.3284$ & $2.2246<R<3.5314$ \\
\hline
\end{tabular}

iii) The nuclear matter state of the outer core of neutron stars: $0.141<\varrho_{N R}<0.235\left(\left(\mathrm{fm}^{-3}\right)\right)$. The corresponding range of $R$ at various values of $m$ are listed in Table 6.

Table 6. The ranges of $\mathrm{R}$ corresponding to the inner crust of neutron stars

\begin{tabular}{|c|c|c|c|}
\hline $\mathbf{m}$ & 0.48 & 0.273 & 0.18 \\
\hline $\mathbf{R}$ & $1.3343<R<1.5701$ & $2.3284<R<2.7606$ & $3.5314<R<4.186927$ \\
\hline
\end{tabular}

iv) The nuclear matter state of the outer crust of neutron stars $\varrho_{N R}<0.141$. The corresponding range of $\mathrm{R}$ at various values of $\mathrm{m}$ are listed in Table 7 .

Table 7. The ranges of $\mathrm{R}$ corresponding to the inner crust of neutron stars

\begin{tabular}{|c|c|c|c|}
\hline $\mathbf{m}$ & 0.48 & 0.273 & 0.18 \\
\hline $\mathbf{R}$ & $R>1.5701$ & $R>2.7606$ & $R>4.186927$ \\
\hline
\end{tabular}

In numerical calculations of the solutions of Type II and Type III, we will vary the value of $\mathrm{R}$ in the above ranges to see in each state of the nuclear matter, what kind of skyrmion exists. 


\section{PHASE TRANSITIONS OF SKYRMIONS OF TYPE III}

In the case the skyrmions of Type III, the control parameters are $m$ and $R$. First, we vary the value of $m$ to find the values of $R$ for the skyrmions of Type III with the topological charge $N=(2 k+1) / 2$.

It is possible to observe that there are ranges of the parameter $R$ and nuclear density where the solution of Type III of a certain integer topological charge. The transition points between the ranges of the integer topological charged solutions are the values of $\mathrm{R}$ where the half-integer skyrmions of Type III exist. We denote the values of $R$ where the skyrmions of the topological $(2 k+1) / 2$ exist as $R_{(2 k+1) / 2}$. The corresponding nuclear densities are denoted as $\varrho_{N R(2 k+1) / 2}^{m}$. The values of $R_{(2 k+1) / 2}$ are found to be independent of the parameter $m$ as listed in Table 8 .

Table 8. The values of the radial parameter R, where the skyrmions of Type III exist

\begin{tabular}{|c|c|c|c|c|}
\hline Topological charge & $\mathbf{m}=\mathbf{0 . 4 8}$ & $\mathbf{m}=\mathbf{0 . 1 8}$ & $\mathbf{m}=\mathbf{0}$ & Notation \\
\hline $\mathbf{N}=\mathbf{1 / 2}$ & 4.373523463472361 & 4.373523463472361 & 4.373523463472361 & $R_{1 / 2}$ \\
\hline $\mathbf{N}=\mathbf{3 / 2}$ & 5.427320890484484 & 5.427320890484484 & 5.427320890484484 & $R_{3 / 2}$ \\
\hline $\mathbf{N}=\mathbf{5 / 2}$ & 6.299784744755655 & 6.299784744755655 & 6.299784744755655 & $R_{5 / 2}$ \\
\hline $\mathbf{N}=\mathbf{7 / 2}$ & 7.054698399436239 & 7.054698399436239 & 7.054698399436239 & $R_{7 / 2}$ \\
\hline $\mathbf{N}=\mathbf{9 / 2}$ & 7.734095198154279 & 7.734095198154279 & 7.734095198154279 & $R_{9 / 2}$ \\
\hline
\end{tabular}

Let us observe that there are certain values of the parameter $R$, where the skyrmions of a certain topological charge exist. Since, in the last section, we have shown that the parameter $R$ is related to the nuclear matter density, the phase transitions occurs at the certain densities. By using Eq. (25), we can calculate the ranges of the nuclear density corresponding to the above values and ranges of $\mathrm{R}$ in Table 9.

Table 9. Values and ranges of the nuclear density corresponding to the solutions of Type III

\begin{tabular}{|c|c|c|c|c|}
\hline $\mathbf{R}$ & $\begin{array}{c}\text { Density } \varrho_{\mathrm{NR}}\left(\mathrm{fm}^{-3}\right) \\
\text { for } \mathbf{m}=\mathbf{0 . 4 8}\end{array}$ & $\begin{array}{c}\text { Density } \varrho_{\mathrm{NR}}\left(\mathrm{fm}^{-3}\right) \\
\text { for } \mathbf{m}=\mathbf{0 . 1 8}\end{array}$ & $\begin{array}{l}\text { Topological } \\
\text { charge }\end{array}$ & Skyrmion \\
\hline$R<R_{1 / 2}$ & $>\varrho_{N R_{1 / 2}}^{0.48}$ & $>\varrho_{N R_{1 / 2}}^{1.18}$ & 0 & No \\
\hline$R_{1 / 2}$ & $\varrho_{N R_{1 / 2}}^{0.48}=0.006523859$ & $\varrho_{N R_{1 / 2}}^{0.18}=0.123711705$ & $1 / 2$ & Yes \\
\hline$R_{1 / 2}<R<R_{3 / 2}$ & $\varrho_{N R_{1 / 2}}^{0.48}>\varrho_{N R}>\varrho_{N R_{3 / 2}}^{0.48}$ & $\varrho_{N R_{1 / 2}}^{0.18}>\varrho_{N R}>\varrho_{N R_{3 / 2}}^{0.18}$ & 1 & No \\
\hline$R_{3 / 2}$ & $\varrho_{N R_{3 / 2}}^{0.48}=0.003413835$ & $\varrho_{N R_{3 / 2}}^{0.18}=0.064736419$ & $3 / 2$ & Yes \\
\hline$R_{3 / 2}<R<R_{5 / 2}$ & $\varrho_{N R_{3 / 2}}^{0.48}>\varrho_{N R}>\varrho_{N R_{5 / 2}}^{0.48}$ & $\varrho_{N R_{3 / 2}}^{0.18}>\varrho_{N R}>\varrho_{N R_{5 / 2}}^{0.18}$ & 2 & No \\
\hline$R_{5 / 2}$ & $\varrho_{N R_{5 / 2}}^{0.48}=0.00218284$ & $\varrho_{N R_{5 / 2}}^{0.18}=0.041393109$ & $5 / 2$ & Yes \\
\hline$R_{5 / 2}<R<R_{7 / 2}$ & $\varrho_{N R_{5 / 2}}^{0.48}>\varrho_{N R}>\varrho_{N R_{7 / 2}}^{0.48}$ & $\varrho_{N R_{5 / 2}}^{0.18}>\varrho_{N R}>\varrho_{N R_{7 / 2}}^{0.18}$ & 3 & No \\
\hline$R_{7 / 2}$ & $\varrho_{N R_{7 / 2}}^{0.48}=0.001554403$ & $\varrho_{N R_{7 / 2}}^{0.18}=0.029476086$ & $7 / 2$ & Yes \\
\hline$R_{7 / 2}<R<R_{9 / 2}$ & $\varrho_{N R_{7 / 2}}^{0.48}>\varrho_{N R}>\varrho_{N R_{9 / 2}}^{0.48}$ & $\varrho_{N R_{7 / 2}}^{0.18}>\varrho_{N R}>\varrho_{N R_{9 / 2}}^{0.18}$ & 4 & No \\
\hline$R_{9 / 2}$ & $\varrho_{N R_{1 / 2}}^{0.48}=0.001179697$ & $\varrho_{N R_{1 / 2}}^{0.18}=0.022370549$ & $9 / 2$ & Yes \\
\hline$R>R_{9 / 2}$ & $<\varrho_{N R_{9 / 2}}^{0.48}$ & $>\varrho_{N R_{9 / 2}}^{0.18}$ & $>5$ & No \\
\hline
\end{tabular}

In Sec. II., we have seen that the integer topological charge solutions of Type III are not skyrmions. The transition points in the parameter $\mathrm{R}$ are where the skyrmions of half integer topological charge $N+1 / 2$ exist. The behavior of the profile function of Eq. (9) near the point 
$F(0)=N \pi$ is similar to the cases of skyrmions of Type I and Type II discussed previously. The trajectories going to different boundary points at $r=0$ are drawn in Fig. 1

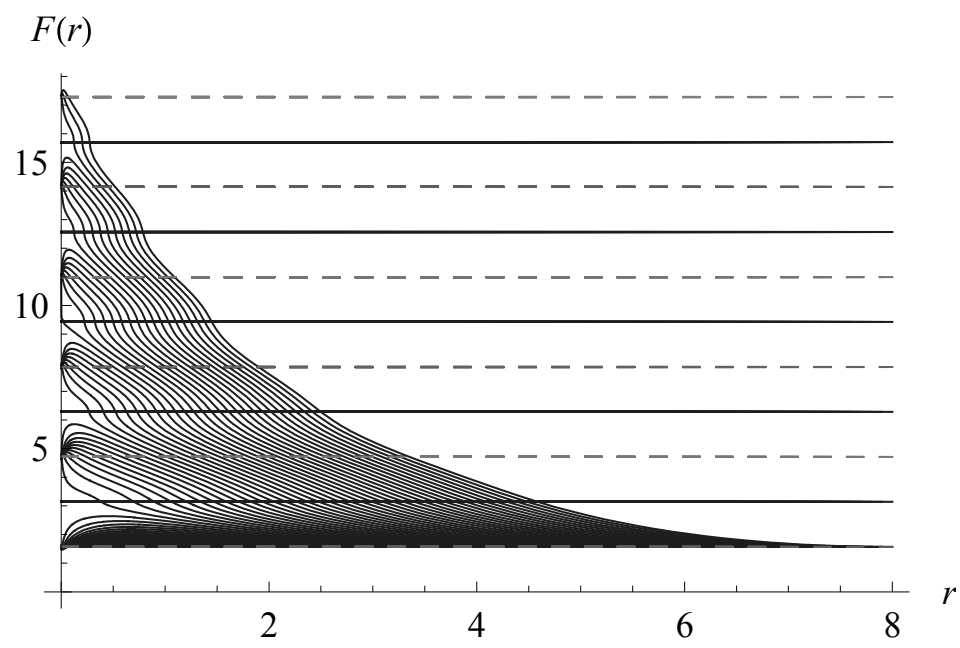

Fig. 1. The profile function trajectories of skyrmions of Type III with $\mathrm{m}=0$

\section{DISCUSSIONS}

In this paper, we study the possibility of modeling the dense nuclear matter by the skyrmions of the Skyrme model in the Wigner-Seitz approximation. In principle, there are three types of skyrmions with integer and half-integer topological charge. We have successfully established a relationship between the range parameter $R$ of the Wigner-Seitz cell and the nuclear matter density to use the skyrmions in different regions inside the neutron stars for the future research.

By using different skyrmion types as a model of the nucleon, we found that the static properties of the modeled nucleon do not fit numerically with a good precision with the experimental values. This result is the motivation to modify the Skyrme model by adding high order interaction terms in the future. In spite of the experimental fit results, we have observed that the mass of the nucleon increases with the increasing nuclear density. This is qualitatively a good agreement with the EMC experiments $[16,17]$.

Additionally, the phase transitions between the skyrmions of Type III with different halfinteger topological charges can also be experimentally interesting. Our model has implied that the large topological charged skyrmion matter of Type III will turn into the one with lower topological charges when the density is increase for instant by a compression as inside of the neutron stars.

The calculations of the static properties of skyrmions in the modified Skyrme model are under progress [18] 


\section{ACKNOWLEDGMENT}

The work is supported in part by ITI-VNU. I am grateful to Professor I.Lovas for the discussions on the finite radius skyrmions.

\section{REFERENCES}

[1] T. H. R. Skyrme, Nuclear Physics, 31 (1962) 556.

[2] G. S. Adkins, C.R. Nappi and E.Witten, Nuclear Physics B 228 (1983) 552.

[3] G. S. Adkins and C. R. Nappi, Nuclear Physics B 233 (1984) 109.

[4] Nguyen Duy Khanh and Nguyen Ai Viet, Skyrmions Revisited with New Boundary Condition ITI-PRE-2013-1/CS arXiv:1309.1313 [hep-ph](submitted to Journal of Computational Physics).

[5] Nguyen Ai Viet, New Skyrmions in a Modified Skyrme Model(accepted for publication in Journal of Science and Technology) (2014).

[6] Nguyen Ai Viet and Pham Thuc Tuyen, J. Phys. G: Nucl. Part. Phys. 15(1989) 937.

[7] H. Y. Cheung and F. Gursey, Modern Physics Letters A 5(21) (1990) 1685.

[8] A. Jackson, A. D.Jakson, A. S. Goldhaber, G. S. Brown and L. C. Castillo, Phys. Lett. B 154 (1985) 101.

[9] L. Marleau, Phys. Rev. D 45, (1992)1776; L. Marleau, Phys. Rev. D 63 (2001) 036007.

[10] A. P. Balachandran, V. P. Nair, S. G. Rajeev and A. Stern, Phys. Rev. Lett. 49 (1982) 1124.

[11] A. S. Goldhaber and N. S. Manton, Phys. Lett. B 198 (1987) 231.

[12] H. Dong et al., Phys. Rev. C 87 (2013) 054332

[13] T.Morinari, Phys. Rev. B 72 (1-10) (2005) 104502; T Morinari, J. Phys. B: At. Mol. Opt. Phys. 39 (2006) S37; doi:10.1088/0953-4075/39/10/S04

[14] Klebanov, Nucl. Phys. B 262 (1985) 133; M. Kugler Phys. Lett. B 208 (1988) 491.

[15] P. Haensel, A. Y. Potekhin and D. G. Yakovlev, Neutron Stars, Springer, (2007) 12.

[16] J.J. Aubert et al., Phys. Lett. B 123 (1983) 275

[17] J. Arrington et al., J. Phys. Conference Series 69 (2007) 012024

[18] Nguyen Duy Khanh and Nguyen Ai Viet, Static Properties of New Skyrmions in a Modified Skyrme Model (to be published). 